\title{
A Velocity Dealiasing Scheme Based on Minimization of Velocity Differences between Regions
}

\author{
Yue Yuan $(\mathbb{D}$, Ping Wang $(\mathbb{D}$, Di Wang $(\mathbb{D}$, and Junzhi Shi \\ School of Electrical and Information Engineering, Tianjin University, Tianjin 300072, China \\ Correspondence should be addressed to Di Wang; wangdi2015@tju.edu.cn
}

Received 29 September 2019; Revised 16 December 2019; Accepted 6 January 2020; Published 20 February 2020

Academic Editor: Hisayuki Kubota

Copyright (C) 2020 Yue Yuan et al. This is an open access article distributed under the Creative Commons Attribution License, which permits unrestricted use, distribution, and reproduction in any medium, provided the original work is properly cited.

\begin{abstract}
The velocity dealiasing is an essential work of automatic weather phenomenon identification, nowcasting, and disaster monitoring based on radial velocity data. The noise data, strong wind shear, and isolated echo region in the Doppler radar radial velocity data severely interfere with the velocity dealiasing algorithm. This paper proposes a two-step velocity dealiasing algorithm based on the minimization of velocity differences between regions to solve this problem. The first step is to correct aliased velocities by minimizing the sum of gradients in every region to eliminate abnormal velocity gradients between points. The interference of noise data and strong wind shear can be reduced by minimizing the whole gradients in a region. The second step is to dealiase velocities by the velocity differences between different isolated regions. The velocity of an unknown isolated region is determined by the velocities of all known regions. This step improves the dealiasing results of isolated regions. In this paper, 604 volume scan samples, including typhoons, squall lines, and heavy precipitation, were used to test the algorithm. The statistical results and analysis show that the proposed algorithm can dealiase the velocity field with a high probability of detection and a low false alarm rate.
\end{abstract}

\section{Introduction}

Radial velocity data is a kind of observation data of Doppler weather radar, which can be used for wind retrieval, severe wind, and other disasters warning. Aliased velocities often appear in radial velocity data with disastrous weather and hamper the weather forecasters' judgments of weather disasters.

The Doppler effect is used to measure velocity in Doppler weather radar. The maximum unaliased velocity that can be measured is also known as the Nyquist velocity and is marked as $V_{N}$. According to the Nyquist sampling theorem, the velocity is defined as

$$
V_{N}=\frac{\lambda f_{P}}{4}
$$

where $\lambda$ is the wavelength of radar and $f_{P}$ is the pulse repetition frequency. If the real radial velocity $V_{O}$ is not in the $-V_{N}$ to $V_{N}$ range, the measured value of radial velocity $V_{m}$ is given as

$$
V_{m}=V_{o} \pm 2 n V_{N}, \quad n=1,2 \ldots,
$$

where positive integer $n$ is called Nyquist number. The problem of aliased velocity can be solved by hardware and software in two ways. Some researchers have made hardware improvements to reduce aliased velocity [1-3]. For example, the staggered pulse repetition time (staggered $-\mathrm{PRT})[1,2]$ is used to reduce the problem by expanding the interval of unaliased velocity. This technique fails at very high radial velocities. At the same time, in order to effectively filter out ground-clutter echo, a specific PRT is needed on the lowest elevation instead of staggered -PRT [4]. Therefore, it is necessary to use the software method to dealiase the velocity.

In the past 40 years, many scholars have carried out a series of researches on software-based dealiasing algorithms. 
Aliased velocity results in false wind shear close to $2 V_{N}$ in the radial velocity field. Therefore, most algorithms dealiased velocity according to the continuity principle of space and time. Among these algorithms, Ray and Ziegler and Bargen and Brown $[5,6]$ proposed two one-dimensional dealiasing radial velocity methods, respectively. Then, two-dimensional methods along the radial and azimuth appeared [4, 7-15]. And James and Houze [16] developed a four-dimensional method based on radial, azimuth, elevation, and time. Among these methods, two-dimensional dealiasing velocity algorithms are the most widely and deeply studied.

The work in [10] checked whether the measured velocity was an aliased velocity according to the difference between it and a reference velocity value and corrected the aliased velocity. The reference velocity value was determined by the velocity value of one neighbor point or the average velocity value of some neighbor points. If such adjacent velocities were invalid, the ambient wind in the sounding data was taken as the reference velocity. The use of this method was limited by the spatial and temporal resolution sparsity of sounding data. Then, He et al. [14] improved the method of [10] in the aspects of the initial radial selection, noise filtering, execution direction of dealiasing velocity, and error checking. Zhang and Wang [12] proposed a two-dimensional dealiasing velocity algorithm that did not require external data for multiple times. According to the reliability of data, the thresholds from tight to loose were set to dealiase velocity. Some other dealiasing velocity methods calculated reference velocity with velocity azimuth display (VAD) method for dealiasing. The work of [17] proposed an improved velocity azimuth display technology (mod -VAD) that used radial velocity data to obtain the correct ambient wind field. Gong et al. [11] dealiased velocity with the mod -VAD. The advantage of this method was that it was resistant to noise interference. But the VAD had a high requirement for data coverage, and the information of wind field change was often too small to describe local small-scale wind shear. Then, $[4,18]$ improved the VAD-based method to dealiase velocity for the purpose of radar data assimilation in which local small-scale shear regions and some isolated echoes away from radar were also removed. Although [19] achieved the repair and correction of some mesocyclone regions, a certain amount of velocity regions were still removed. The common feature of the above algorithms is that a reference velocity is used to check whether a detail in the radial velocity field is aliased. This reference velocity is derived either from the mean velocity of the neighbor points or from other data (such as sounding data) or VAD results. Therefore, these can be generalized as "point to block" comparison methods.

The work in [7] first proposed a two-dimensional dealiasing velocity algorithm, in which the velocity field was divided into different regions, and checking and correction of data were carried out according to the boundary of the regions. Considering the influence of data noise, [20] transformed the problem of dealiasing velocity into solving the extremum problem of linear equations based on the hypothesis of a smooth velocity field. The work of [13] optimized the weight factors in the linear equations of [20].
And these methods used sounding data, VAD wind field information, or wind speed in radar main echo region to correct velocity in isolated echo region. In essence, these kinds of algorithms used the "block to block" comparison method to dealiase velocity. Compared with the "point to block" method, the "block to block" method has a stronger antinoise ability. One of the deficiencies is that in the case of strong shear. If the radial velocity differences in the boundary of adjacent regions are higher than the Nyquist velocity $V_{N}$, it may lead to false dealiasing.

In this paper, the "block to block" method is used to dealiase velocity. According to the continuity of the wind field, a method named minimization of velocity differences between regions (MVDR) is proposed for velocity dealiasing. First, the velocities in each isolated region were dealiased. All abnormal wind shear points close to $2 V_{N}$ in an isolated region were found, and the region containing the abnormal velocity gradient was divided into multiple subregions. The velocity dealiasing was carried out by using the principle of minimizing the sum of gradients in every region. Next, to solve the aliased problems existing between isolated regions, this paper designed a regional velocity check model with strong antinoise ability according to the continuity principle of velocity in radial and azimuth direction. The model comprehensively considered factors such as velocity differences and distances between the unchecked regions and the adjacent checked regions to make a judgment on whether it is aliased or not. There are two main advantages of this algorithm. (1) In the case of dealiasing in a connected region, our algorithm proposes a step to distinguish abnormal wind shear and to reduce the strong shear region being dealiased incorrectly. (2) Our algorithm designs a dealiasing model for isolated regions away from the radar to improve the dealiasing performance of these regions.

The arrangement of the paper is as follows. Section 2 introduces the data used in this article. Section 3 describes in detail the methods proposed in this paper, including intraregional dealiasing and interregional dealiasing. Section 4 compares the statistical results of different dealiasing velocity algorithms in various weather conditions and analyzes the experimental results. Section 5 makes a summary and prospect of the work of this paper.

\section{Data}

In this paper, the radial velocity data was from some S-band radars belonging to China New Weather Generation Radar (CINRAD) network. These radars were mainly located along with coastal regions in China. The wavelength of the S-band radar was about $10 \mathrm{~cm}$, the radial resolution was about $250 \mathrm{~m}$, and the azimuth resolution was about $1^{\circ}$. The Volume Coverage Pattern 21 (VCP21) scan mode was selected. In this mode, a volume scan file was generated about every 6 minutes, and each volume scan file contains nine elevation angles $\left(0.5,1.5,2.4,3.4,4.3,6.0,9.9,14.6\right.$, and $\left.19.5^{\circ}\right)$. In the lower six elevation angles, the maximum unambiguous velocity was about $27 \mathrm{~ms}^{-1}$. And in the three higher elevation angles, the maximum unambiguous velocity was about $32 \mathrm{~ms}^{-1}$. 
The algorithm test sample set was composed of 604 volume scan data in different weather types, including a total of 50 volumes of the two typhoon processes, a total of 123 volumes of the four squall line processes, and a total of 431 volumes from seven heavy precipitation processes. The characteristics of velocity data of these kinds of weather were as follows. The velocity value of typhoons was relatively large, and aliased velocities in the radial velocity field were easy to appear, sometimes even appearing "double aliasing". The squall line was prone to strong shear in the radial velocity field. Aliased velocities were often found in convective weather data caused by heavy precipitation. In addition, range folding and various noises in the radial velocity field of radar also interfered with the dealiasing algorithms, increasing the difficulty of dealiasing. In each of the 604 samples, at least one elevation angle presented aliased velocities.

The percentage of aliased data in all valid data was $5.3 \%$. However, in the most severe elevation angle of each sample, the average percentage of aliased data to valid data was as high as $12.0 \%$. The reference velocity field was determined by the manual check, which was used to evaluate the automatic dealiasing algorithm. When manually editing, reference was made to the lower and higher tilt in current radial velocity volume and the corresponding tilt in previously dealiased velocity volume. Besides, the manually dealiased velocity field was also checked by the European Centre for MediumRange Weather Forecasts (ECMWF) Re-Analysis (ERAInterim). In difficult cases, where it was not clear whether there was an error or what was the correct solution, the data was not included. These data only accounted for $0.05 \%$ percent of all valid data and $1.5 \%$ percent of aliased data.

\section{Methodology}

The proposed algorithm is divided into two steps: the first step is dealiasing in every region, and the second step is dealiasing between interregions. Jing and Wiener [20] pointed out that, in the case of strong wind shear, the dealiasing was likely to fail. In the process of dealiasing in a region, we add a substep of seeking points with the abnormal gradient. Only if there are abnormal gradients between two adjacent points, there would be aliased velocities in this region. This reduces the chance that strong wind shear will be mistaken for velocity folds. The isolated region far from the radar is always a difficulty in velocity dealiasing. In some cases, these regions cannot find the reference points in a small window. Therefore, in the second step, according to the principle that the velocities of points close to each other are similar (wind shear will also be considered), we seek the reference velocity from the dealiased velocity within a certain range and judge whether the velocity in this region is aliased.

3.1. Dealiasing in Every Region. As discussed above, if there is a velocity difference approaching $2 V_{N}$ in a region, it is generally considered that velocity ambiguity appears. Therefore, firstly, the velocity differences between each point and its adjacent points are calculated, and the potential aliased velocity points can be found. Then, this region can be divided into subregions according to discontinuous velocity. Last, the aliased velocities are corrected according to the principle of minimizing the velocity gradients between subregions. In the face of $n_{r}$ isolated regions in a radial velocity field, Figure 1 shows the flow chart of implementing the above algorithm, which mainly includes the steps of calculating the gradients of points in the region, seeking the points with abnormal gradient, obtaining subregions by region growth method and checking aliased velocities in the region The number of isolated regions $n_{r}$ is automatically determined, and it is the number of valid data regions surrounded by missing data.

3.1.1. Calculating the Gradients of Points and Seeking the Abnormal Gradients. For any point $p$ within the connected region $\Omega$, its neighborhood domain $N(p)$ is defined as

$$
N(p)=\{q \mid d(p, t)=1, t \in \Omega\},
$$

where $d(p, t)$ is the distance measurement between point $p$ and point $t$, and the neighborhood distance threshold $n$ is 1 . Figure 2 shows the four neighbors of a point, and the gray points are the neighborhoods of the black point. It should be pointed out that the format of the original radar data is discrete 3D polar coordinates. The three-dimensional coordinates of a point are elevation $\alpha$, radial value $r$, and azimuth value $\theta$, respectively. So, the points $p$ and $q$ can be marked as $\left(\alpha, r_{p}, \theta_{p}\right)$ and $\left(\alpha, r_{q}, \theta_{q}\right)$, respectively. In order to maintain the simplicity and efficiency of the algorithm, we use the following method instead of geometric distance when calculating the distance between two points in this paper. Two adjacent points with the same $a$ and $r$ and two adjacent points with the same $a$ and $\theta$ are defined as 1-unit distance. Then, if any two points $p$ and $q$ are in the same elevation angle, the distance between them is defined as

$$
\begin{aligned}
& d(p, q)=\sqrt{d_{r}^{2}(p, q)+d_{\theta}^{2}(p, q)} \\
& d_{r}(p, q)=\left|r_{p}-r_{q}\right| \\
& d_{\theta}(p, q)=\min \left(\left|\theta_{p}-\theta_{q}\right|, 360^{\circ}-\left|\theta_{p}-\theta_{q}\right|\right),
\end{aligned}
$$

where $d_{r}(p, q)$ is the radial distance between points $p$ and $q$, and $d_{\theta}(p, q)$ is the azimuth distance between points $p$ and $q$.

Assume that the radial velocity value of point $p$ is $v_{p}$. Since it is a unit distance away from the neighbor points, the gradient from point $p$ to its neighborhood can be defined as

$$
\operatorname{grad}(p)=\max \left\{\left|v_{t}-v_{p}\right| / d(p, t), t \in N(p)\right\} .
$$

The gradient and velocity difference between two adjacent points are numerically equivalent. Thus, the gradient of point $p$ is also equivalent to

$$
\operatorname{grad}(p)=\max \left\{\left|v_{t}-v_{p}\right|, t \in N(p)\right\}
$$




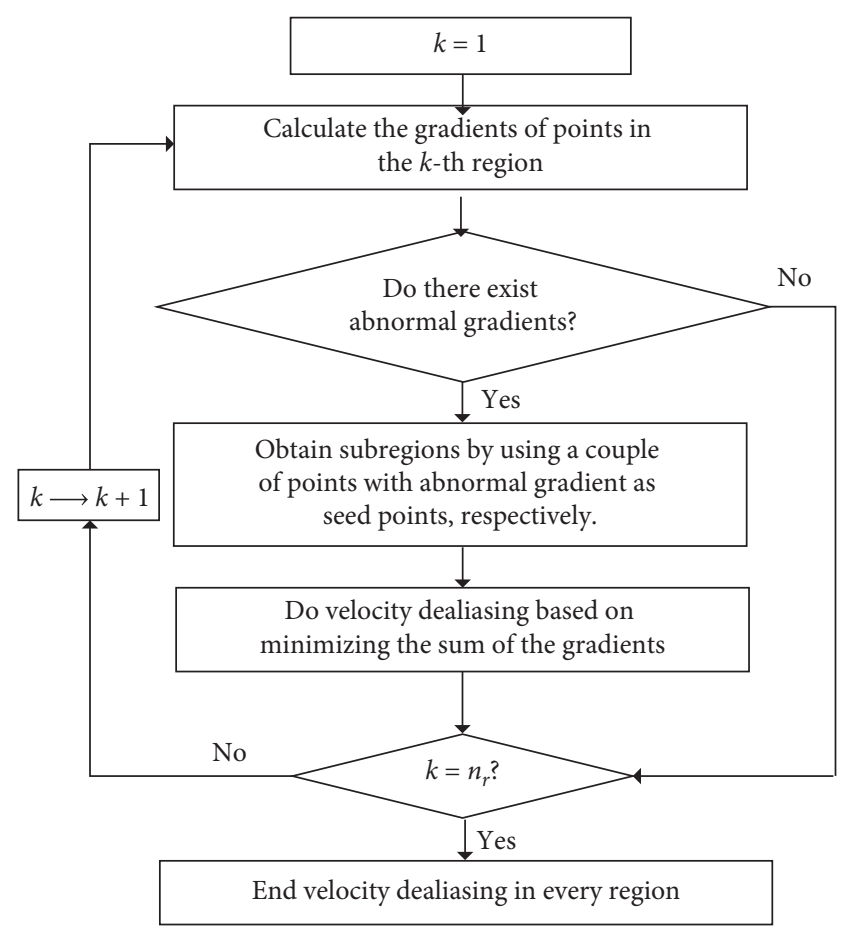

FIGURE 1: The flow chart of velocity dealiasing in every region in each elevation angle.

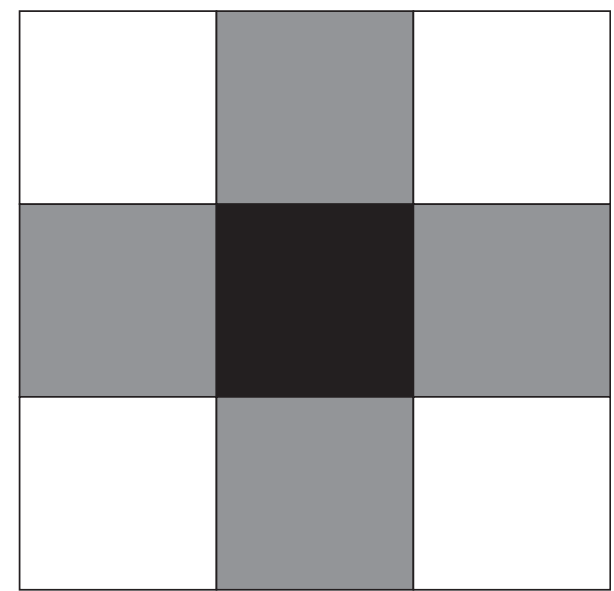

FIGURE 2: Four neighborhoods of a point. The four gray points are neighborhoods of the black point.

According to the characteristics of aliased velocities in the image, if $\operatorname{grad}(p)$ is near to $2 V_{N}$, one of the points $p$ and $q$ is generally an aliased point. In this paper, these two points are specially called a couple of points with an abnormal gradient $(p, q)$. Therefore, the set of a couple of points with the abnormal gradient $P$ in the region $\Omega$ can be get by

$$
P=\left\{(p, q) \mid \operatorname{grad}(p)>G_{1}, q \in N(p), p \in \Omega\right\} .
$$

Theoretically, $G_{1}$ is a threshold close to $2 V_{N}$. If the elements in set $P$ are empty, it is considered that there is no aliased velocity in this region, and the next region can be checked. If the elements in the set $P$ are not empty, it is necessary to perform some operations such as subregion segmentation and dealiasing.

3.1.2. Subregion Segmentation. Each couple of points with the abnormal gradient $(p, q)$ is taken as two seed points for region growing to obtain the subregions $S_{p}$ and $S_{q}$, respectively. Take $S_{p}$ as an example to illustrate the region growing method. The initial element of $S_{p}$ is only point $p$, and $S_{p}$ is generated as the following two steps.

Step (1). With $p$ as the seed point, all points $t$ satisfying the condition of Equation (8) are added to $S_{p}$ :

$$
\left|v_{t}-v_{p}\right| \leq \delta, \quad t \in N(p), t \notin S_{p} .
$$

Step (2). Take the point $t$ in Step (1) as the new seed point and repeat Step (1) until no new point satisfies Equation (8).

The parameter $\delta$ in Equation (8) should be a threshold slightly greater than zero. Referring to the growing threshold of [9], we set $\delta=5 \mathrm{~ms}^{-1}$. In this method, the radar echo with a slow change in velocity is divided into a subregion, as shown in Figure 3. There are small velocity differences between adjacent points in a subregion. But the maximum and minimum velocity values in the region may vary significantly. In [9], only the points with similar velocities are divided into a subregion, and the sector in Figure 3 may be divided into several subregions. In a similar way, we can get $S_{q}$. Therefore, the region $\Omega$ is segmented into two subregions $S_{p}$ and $S_{q}$ according to the "point pair" $(p, q)$.

3.1.3. Dealiasing in a Connected Region. Because there are abnormal gradients between $S_{p}$ and $S_{q}$ subregions, it can be considered that aliased velocities appear in one of them. For the convenience of discussion and analysis below, we suppose that $v_{p}>v_{q}$. Then, two hypothesizes can be made. Hypothesis 1 . The subregion $S_{p}$ is the region with aliased velocities. In this assumption, all points in $S_{p}$ need to be dealiased (add $2 V_{N}$ ), and the dealiased subregion can be marked as $S_{p}^{\prime}$. Hypothesis 2. The subregion $S_{q}$ is the region with aliased velocities. In this assumption, all points in the $S_{q}$ need to be dealiased (subtract $2 V_{N}$ ), and the dealiased subregion can be marked as $S_{q}^{\prime}$.

Obviously, $S_{p}^{\prime}$ and $S_{p}$ represent the same region, but the velocities of the points are different. And it is similar to $S_{q}^{\prime}$ and $S_{q}$. The sum of the gradients in the region $\Omega$, denoted by $T_{o}$, can be written as

$$
T_{o}=\sum_{t} \operatorname{grad}(t), \quad t \in \Omega
$$

Suppose that these points not belonging to $S_{p}$ and $S_{q}$ in the region $\Omega$ belong to the subregion $S_{c}$. Then, the sum of gradients $T_{p}$ of this region after dealiasing velocity in $\mathrm{Hy}-$ pothesis 1 is

$$
T_{p}=\sum_{t} \operatorname{grad}(t), \quad t \in S_{p}^{\prime} \cup S_{q} \cup S_{c} .
$$




\begin{tabular}{|c|c|c|c|c|c|c|c|}
\hline 27 & 23 & 20 & 18 & 16 & 12 & 8 & 5 \\
\hline 26 & 22 & 22 & 18 & 15 & 11 & 7 & 4 \\
\hline \hline 27 & 21 & 24 & 20 & 16 & 12 & 8 & 4 \\
\hline
\end{tabular}

FIgURE 3: A hypothetical sector is divided into a subregion in our segmentation method.

The sum of gradients $T_{q}$ of this region after dealiasing velocity in Hypothesis 2 is

$$
T_{q}=\sum_{t} \operatorname{grad}(t), \quad t \in S_{q}^{\prime} \cup S_{p} \cup S_{c} .
$$

If Hypothesis 1 or Hypothesis 2 is right, the abnormal gradients can be eliminated. In order to determine which hypothesis is right, the principle of minimizing the velocity differences between subregions is established. That is the sum of gradients of the correctly dealiasing region is less than $T_{o}$, and the sum of gradients of the incorrectly dealiasing region is greater than $T_{o}$. It is embodied in the following four kinds of situations. Case 1. If $\min \left\{T_{o}, T_{p}, T_{q}\right\}=T_{p}$, and $T_{q}>T_{o}$, Hypothesis 1 is considered to be correct, and $S_{p}$ is replaced by $S_{p}^{\prime}$. Case 2. If $\min \left\{T_{o}, T_{p}, T_{q}\right\}=T_{q}$, and $T_{p}>T_{o}$, Hypothesis 2 is considered to be correct, and $S_{q}$ is replaced by $S_{q}^{\prime}$. Case 3. If $\min \left\{T_{o}, T_{p}, T_{q}\right\}=T_{o}$, Hypothesis 1 and Hypothesis 2 are overridden, and the region $\Omega$ remains unchanged. Case 4. If $\max \left\{T_{p}, T_{q}\right\}<T_{o}$, it is difficult to determine which subregion is aliased directly. We temporarily assume that the region with fewer points is aliased. In this way, the abnormal gradients in the region $\Omega$ can be eliminated. And whether the dealiasing in Case 4 is correct or not will be left to the subsequent interregional dealiasing.

Figure 4 illustrates the behavior of the dealiasing in a region in a hypothetical azimuth-range sector. In this example, the value of $V_{N}$ is $27 \mathrm{~ms}^{-1}$, and the value of $G_{1}$ is $1.5 V_{N}$, which is $40.5 \mathrm{~ms}^{-1}$. Figure $4(\mathrm{a})$ is the raw radial velocity. Figure $4(\mathrm{~b})$ is the gradient of every point in Figure 4(a) calculated by Equation (5). The red rectangles in Figure 4(b) mark four couples of points with abnormal gradient whose gradients are greater than $40.5 \mathrm{~ms}^{-1}$. The red rectangles are also shown at the corresponding positions in Figure 4(a). Then, in this sector, there are four elements in the set of a couple of points with abnormal gradient $P$. The "velocity pairs" corresponding to these four elements are $(-25,26),(-27,27),(-26,26)$, and $(-26,24)$. As described above, after the substep of seeking the couples of points with abnormal gradient, two subregions are segmented based on a couple of points. According to the region growing method, the subregions are segmented from the two points with velocity values of $-25 \mathrm{~ms}^{-1}$ and $26 \mathrm{~ms}^{-1}$, respectively. The results of the segmentation are denoted by the blue polygons in Figure 4(a). The velocity in one of the subregions mark by blue polygons should be aliased. Figures 4(c) and 4(d) show the dealiased velocity under Hypothesis 1 and Hypothesis 2, respectively. According to the calculation, the sum of gradients of the raw velocity and dealiased velocity under the two hypothesizes (corresponding to Figures 4(a), 4(c), and 4(d), respectively) is 504, 293, and 109, respectively. Because Case 4 is met, Figure 4(d) is considered as the dealiasing result of this time. Then, the dealiasing is continued in the velocity in Figure 4(d). It is found that the other three couples of points with the abnormal gradient in set $P$ no longer exist. Thus, there is no abnormal gradient in the sector, and the Figure 4(d) is the final result of the intraregional dealiasing in the sector.

3.2. Dealiasing between Interregions. Generally speaking, the large velocity echo covering or near the radar station is called the main velocity echo. The average velocity of this main region is usually between $\left[-V_{N}, V_{N}\right][8,20]$. Thus, the aliased velocities in the main velocity region can be corrected by the method in Section 3.1. However, sometimes, the isolated velocity region far from the radar station is aliased, e.g., regions similar to Case 4 in Section 3.1.3 and regions with all aliased velocities. In these cases, it is necessary to seek available velocity information from the adjacent regions to get the velocity difference between the two regions for dealiasing. The velocity difference between the two regions can be determined by the velocity difference between the boundary points of the two regions. Then, two strategies are developed, namely, the strategy of assigning a large weight to the region with a short distance and high confidence, and the strategy of dealiasing from the region close to the radar to the region far away from the radar. Suppose that the number of regions need to be executed is $n_{a}$, and these regions are sorted in ascending order according to the distance to the radar. Then, the flow chart of velocity dealiasing between regions is shown in Figure 5, which mainly includes determining the reference point of the region, calculating the velocity difference and its weight between the region and the reference point, and dealiasing between interregions. The number of isolated regions $n_{a}$ is automatically determined, and it is the number of valid data regions surrounded by missing data.

3.2.1. Determination of Regional Reference Points. Suppose that there are $m$ dealiased regions denoted by $\Omega_{i}(i=1,2, \ldots, m)$, respectively, and the next region will be checked named as $\Omega_{m+1}$. The region $\Omega_{m+1}$ is segmented into several subregions by region growing method in Section 3.1.2 and $l$ subregions are obtained named as $S_{j}(j=$ $1,2, \ldots, l)$, respectively. Assuming that point $q$ is a boundary point of the region $\Omega_{i}$, all boundary points of the region $S_{j}$ directly connected with point $q$ compose the set $B\left(S_{j}, q\right)$, which can be expressed by Equation (12):

$$
\begin{aligned}
B\left(S_{j}, q\right) & =\left\{p \mid L_{p q} \cap\left(S_{j} \cup\left(\underset{t \in(\{1, \ldots, m+1\})}{\cup} \Omega_{t}\right)\right)\right. \\
& \left.=\{p, q\}, p \in S_{j}, S_{j} \subset \Omega_{m+1}\right\},
\end{aligned}
$$

where $L_{p q}$ represents the set of points on the line segment connected by point $p$ and point $q$. Equation (12) indicates that $L_{p q}$ intersects these regions only at two points $p$ and $q$. If the set $B\left(S_{j}, q\right)$ is empty, point $q$ is not the reference point of subregion $S_{j}$. If the set $B\left(S_{j}, q\right)$ is not empty, the point $q$ is a reference point of subregion $S_{j}$, and region $\Omega_{i}$ is a reference 


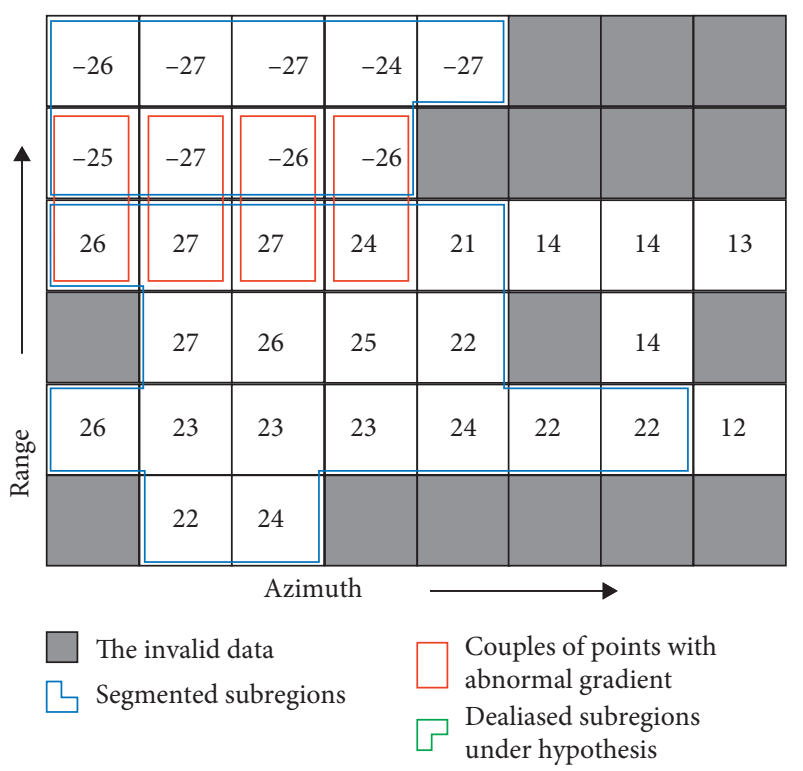

(a)

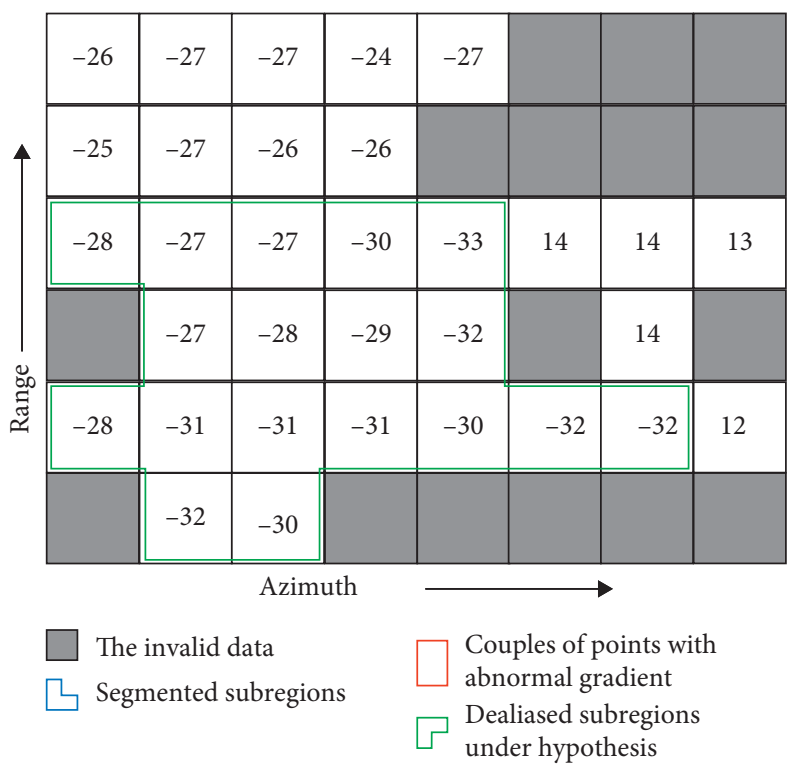

(c)

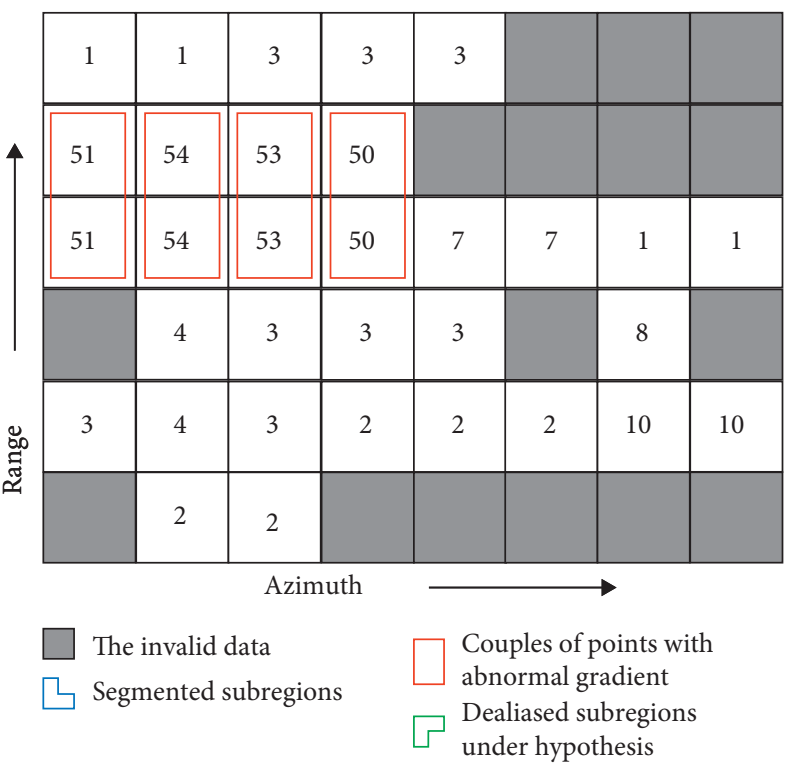

(b)

\begin{tabular}{|c|c|c|c|c|c|c|c|}
\hline 28 & 27 & 27 & 30 & 27 & & & \\
\hline 29 & 27 & 28 & 28 & & & & \\
\hline \multirow[t]{2}{*}{26} & 27 & 27 & 24 & 21 & 14 & 14 & 13 \\
\hline & 27 & 26 & 25 & 22 & & 14 & \\
\hline \multirow[t]{2}{*}{26} & 23 & 23 & 23 & 24 & 22 & 22 & 12 \\
\hline & 22 & 24 & & & & & \\
\hline
\end{tabular}

The invalid data

Segmented subregions

Couples of points with abnormal gradient Dealiased subregions under hypothesis

(d)

FIGURE 4: The behavior of the dealiasing in a region illustrated within a hypothetical azimuth-range sector: (a) the raw velocity, (b) the gradient corresponding to the raw velocity, (c) the dealiased velocity under Hypothesis 1 , (d) the dealiased velocity under Hypothesis 2 . Gray points indicate invalid data. Blue polygons in (a) mark the segmented subregions. Red rectangles in (a) and (b) denote the couples of points with abnormal gradient. Green polygons in (c) and (d) mark the dealiased subregions under different hypothesizes, respectively.

region of subregion $S_{j}$. The point closest to point $q$ in $B\left(S_{j}, q\right)$ is named as $p_{m}$ :

$$
p_{m}=\underset{t}{\arg \min } d(t, q), \quad t \in B\left(S_{\mathrm{j}}, q\right) .
$$

Then, the distance between point $q$ and subregion $S_{j}$ is defined as

$$
d\left(q, S_{j}\right)=d\left(q, p_{m}\right)
$$

The velocity difference between point $q$ and point $p_{m}$ is defined as the velocity difference between point $q$ and $S_{j}$ :

$$
\Delta v\left(q, S_{j}\right)=v_{q}-v_{p_{m}}
$$

3.2.2. Determining the Weights of Velocity Differences. The checked regions that are closer to the unchecked region and at a high confidence level should show a greater impact on the unchecked region. Simply speaking, the weight of point $q$ in the region $\Omega_{i}$ to the subregion $S_{j}$ is shown in Equation (16):

$$
I\left(q, S_{j}\right)=I_{d}\left(q, S_{j}\right) I_{s}(q)
$$




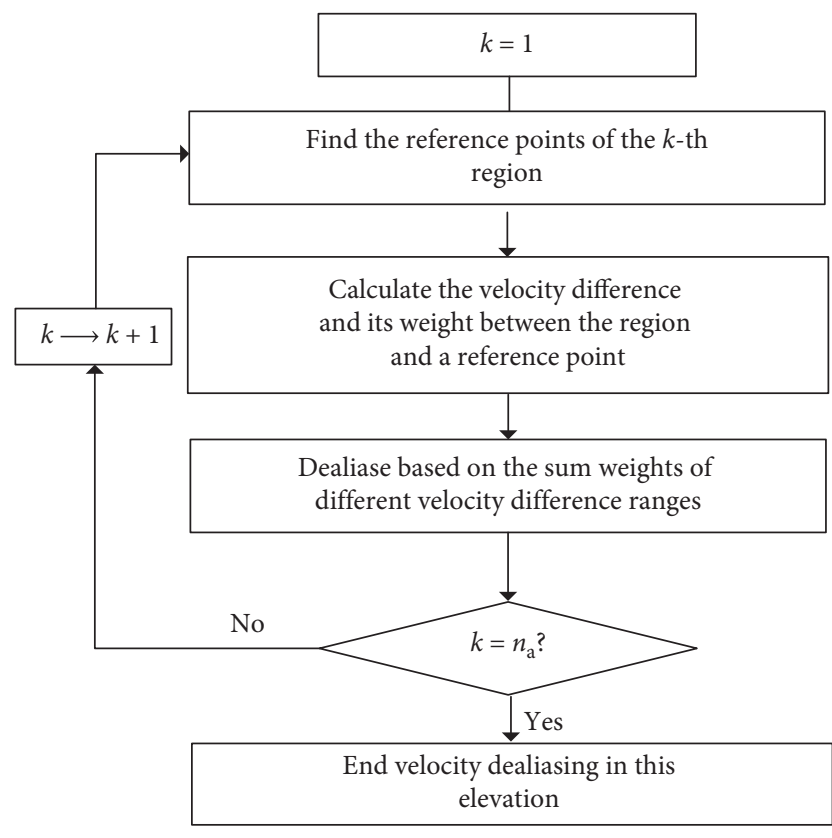

FIGURE 5: The flow chart of velocity dealiasing between interregions in each elevation angle.

where $I_{d}\left(q, S_{j}\right)$ represents the weight related to the distance between point $q$ and subregion $S_{j}$, and $I_{s}(q)$ represents the confidence weight factor of the velocity of point $q$. In this definition, two factors, distance and confidence, can be calculated separately. The design of distance weighting factor $I_{d}\left(q, S_{j}\right)$ is as follows:

$$
I_{d}\left(q, S_{j}\right)= \begin{cases}\frac{1}{d\left(q, S_{j}\right)}, & d_{r}\left(p_{m}, q\right)<\rho, d_{\theta}\left(p_{m}, q\right)<\lambda, \\ 0, & \text { otherwise, }\end{cases}
$$

where $\rho$ and $\lambda$ are the thresholds of radial distance and azimuth distance, respectively. And the default values of the parameters $\rho$ and $\lambda$ are $80 \mathrm{~km}$ and $15^{\circ}$, respectively. The velocity value in a large region generally shows a high degree of confidence. Therefore, the confidence weight factor $I_{s}(q)$ is designed to be related to the size of the region where $q$ is located. $I_{s}(q)$ is calculated by using Equation (18):

$$
I_{s}(q)=\sqrt{\operatorname{card}\left(\Omega_{i}\right)}, \quad q \in \Omega_{i},
$$

where $\operatorname{card}(T)$ represents the number of points of the set $T$.

3.2.3. Dealiasing between Regions. Suppose that subregion $S_{j}$ is a region that needs to be checked. There may be several reference regions of the subregion $S_{j}$. And there may be several points in every reference region that are the reference points of the subregion $S_{j}$. First, the velocity difference $\Delta v\left(q, S_{j}\right)$ between each reference point $q$ and regional $S_{j}$ is divided into three intervals. The three velocity difference intervals are $\left(-\infty,-G_{2}\right),\left[-G_{2}, G_{2}\right]$, and $\left(G_{2}, \infty\right)$. The sum of weights corresponding to the three different intervals is named as $C_{I+}$,
$C_{I 0}$, and $C_{I-}$, respectively, and their initial values are all 0 . Then, the weight $I\left(q, S_{j}\right)$ is added to different sums of weights according to the velocity difference $\Delta v\left(q, S_{j}\right)$. Last, to be checked subregion $S_{j}$ is determined by the values of the sum of weights $C_{I+}, C_{I 0}$, and $C_{I-}$. The specific operation is as follows.

Step (1). For each reference point $q, q \in \Omega_{i}$, $i=1,2, \ldots l, l \leq m$, do the following calculation:

$$
\begin{cases}C_{I+}=C_{I+}+I\left(q, S_{j}\right), & \Delta v\left(q, S_{j}\right)>G_{2}, \\ C_{I-}=C_{I-}+I\left(q, S_{j}\right), & \Delta v\left(q, S_{j}\right)<-G_{2}, \\ C_{I 0}=C_{I 0}+I\left(q, S_{j}\right), & \text { otherwise, }\end{cases}
$$

where $G_{2}$ is the threshold of velocity dealiasing between interregions.

Step (2). If $C_{I+}>C_{I 0}$ and $C_{I+}>C_{I-}$, it is necessary to dealiase the velocities in the subregion $S_{j}$, and $2 V_{N}$ should be added to the velocity value of every point in the subregion $S_{j}$. If $C_{I-}>C_{I 0}$ and $C_{I-}>C_{I+}$, it is also necessary to dealiase the velocities in the subregion $S_{j}$, and $2 V_{N}$ should be subtracted from the velocity of every point in the subregion $S_{j}$. Otherwise, the subregion $S_{j}$ remains unchanged.

Figure 6 illustrates the behavior of the dealiasing between interregions in a hypothetical azimuth-range sector. In this example, the value of $V_{N}$ is $27 \mathrm{~ms}^{-1}$, and the value of $G_{2}$ is $1.3 V_{N}$, which is $35.1 \mathrm{~ms}^{-1}$. The regions $\Omega_{1}, \Omega_{2}$, and $\Omega_{3}$ are three isolated regions from the bottom to the top in Figure 6 . Among them, the regions $\Omega_{1}$ and $\Omega_{2}$ have been checked by the dealiasing between interregions, and the region $\Omega_{3}$ is to be checked. The region $\Omega_{3}$ can be segmented into two subregions $S_{1}$ and $S_{2}$, as indicated by the blue rectangles. According to the above, the reference points of $S_{1}$ are first found. There are three points in $\Omega_{1}$ as reference points of subregion $S_{1}$ (green boxes on the lower side of Figure 6(a)). These three points are connected to the nearest point in the subregion $S_{1}$ with blue lines. Then, the velocity differences between these three points and subregion $S_{1}$ are calculated as 51,49 , and $49 \mathrm{~ms}^{-1}$, respectively. The red boxes in the region $\Omega_{1}$ mark several nonreference points of subregion $S_{1}$. According to Equation (13), since the point with a velocity of $25 \mathrm{~ms}^{-1}$ marked by the red box in Figure 6(a) cannot be directly connected to any boundary point in the subregion $S_{1}$ (the black dashed line will cross the points in the region $\Omega_{2}$ ), this point is not a reference point of subregion $S_{1}$. There are also two points in $\Omega_{2}$ that are the reference points of subregion $S_{1}$ (two green boxes in the middle of Figure 6(a)), and their velocity differences with $S_{1}$ are 29 and $26 \mathrm{~ms}^{-1}$, respectively. In Figure 6(a), the sum of weights corresponding to the three different velocity difference intervals named as $C_{I+}, C_{I 0}$, and $C_{I-}$ is $2.22,1.41$, and 0 , respectively. According to the described above, it is necessary to add $2 V_{N}$ to the velocity value of each point in the subregion $S_{1}$, and the result is shown in Figure 6(b). Next, the dealiasing between interregions is performed on the subregion $S_{2}$ in the region $\Omega_{3}$. Similarly, the points marked in the green boxes in Figure 6(b) are the reference points of subregion $S_{2}$; these reference points 


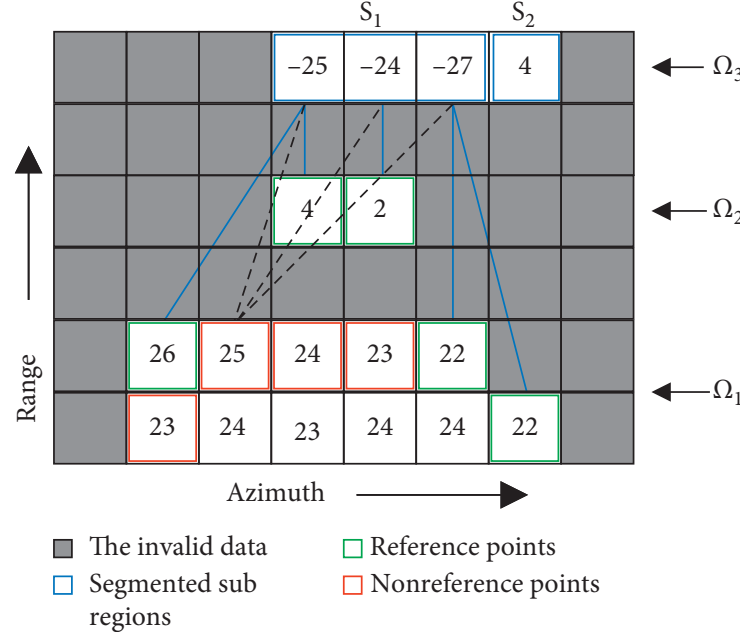

(a)

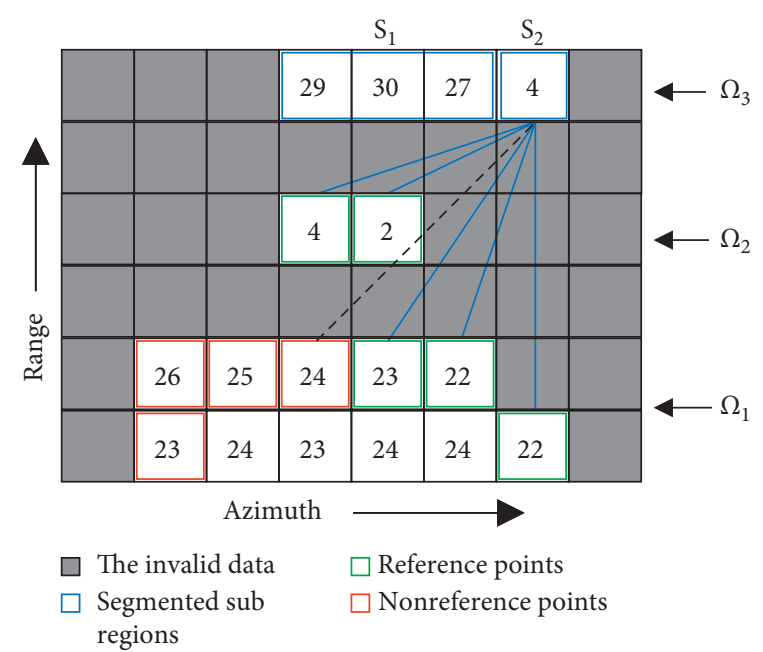

(b)

Figure 6: The behavior of the dealiasing between interregions illustrated within a hypothetical azimuth-range sector. (a) The behavior of seeking reference points of subregion $S_{1}$. (b) The behavior of seeking reference points of subregion $S_{2}$ after the dealiasing of the subregion $S_{1}$. Gray points indicate invalid data. $\Omega_{1}, \Omega_{2}$, and $\Omega_{3}$ represent three isolated regions from the bottom to the top in the sector. Among them, the regions $\Omega_{1}$ and $\Omega_{2}$ have been checked by dealiasing between interregions, and the region $\Omega_{3}$ is to be checked. The region $\Omega_{3}$ can be segmented into two subregions $S_{1}$ and $S_{2}$, as indicated by the blue rectangles. The blue line indicates that the two points can be directly connected, and the black dashed line indicates that the two points cannot be directly connected. The points marked in the green boxes in (a) are the reference points of the subregion $S_{1}$; the blue line connects the reference point to the nearest point in the subregion $S_{1}$, and the red boxes mark several points that are not the reference points of the subregion $S_{1}$. Similarly, the points marked in the green box in (b) are the reference points of the subregion $S_{2}$; the blue line connects the reference point to the nearest point in the subregion $S_{2}$, and the red boxes mark several points that are not the reference points of the subregion $S_{2}$.

are connected to the nearest point in the subregion $S_{2}$ with blue lines. After calculation, the velocity in Figure 6(b) is the final result of the dealiasing between interregions in this sector.

\section{Algorithm Testing}

In order to evaluate the performance of the dealiasing method proposed in this paper, a comparative experiment with the improved dealiasing algorithm (CIDA) proposed by [14] for CINRAD is tested. And the probability of detection (POD), the false alarm rate (FAR), and the critical success index (CSI) were used to evaluate the performance of the automatic dealiasing algorithm. The manually edited velocity fields described in Section 2 were used as the true velocity fields for testing these two automatic dealiasing algorithms. The results of automatic dealiasing were compared with the manually dealiased velocity fields. The number of points in the radial velocity field where the aliased data was correctly dealiased was denoted by $W$, the number of points where the aliased data was not correctly dealiased was denoted by $X$, and the number of points where the normal data was wrongly dealiased was denoted by $Z$. The calculation formulas of POD, FAR, and CSI are as follows:
TABLE 1: Comparative experiment results of different velocity dealiasing algorithms.

\begin{tabular}{|c|c|c|c|c|}
\hline & Weather type & POD (\%) & FAR (\%) & CSI (\%) \\
\hline \multirow{4}{*}{ MVDR } & Typhoon & 98.43 & 1.00 & 97.46 \\
\hline & Squall line & 96.90 & 0.82 & 96.13 \\
\hline & Heavy rain & 99.07 & 0.19 & 98.88 \\
\hline & All & 98.87 & 0.35 & 98.53 \\
\hline \multirow{4}{*}{ CIDA } & Typhoon & 96.24 & 3.82 & 92.70 \\
\hline & Squall line & 91.24 & 1.69 & 89.84 \\
\hline & Heavy rain & 97.20 & 0.37 & 97.54 \\
\hline & All & 97.05 & 1.00 & 96.10 \\
\hline \multicolumn{5}{|c|}{ POD $-W$} \\
\hline \multicolumn{5}{|c|}{$\mathrm{POD}=\overline{W+X}$} \\
\hline \multicolumn{5}{|c|}{$Z$} \\
\hline \multicolumn{5}{|c|}{$\mathrm{FAR}=\overline{W+Z}$} \\
\hline \multicolumn{5}{|c|}{$W$} \\
\hline
\end{tabular}

In this evaluation, radar data was categorized into three classes according to different weather types: typhoons, squall lines, and heavy rain. There were 604 volume scans in 13 
weather processes of three different types used to test the two dealiasing algorithms. Table 1 showed the evaluation results when the thresholds of $G_{1}$ and $G_{2}$ are selected as $1.5 V_{N}$ and $1.3 V_{N}$, respectively. The following can be seen:

(1) In the experiment, the POD, FAR, and CSI values of the MVDR algorithm were $98.87 \%, 0.35 \%$, and 98.53\%, respectively. All POD values and CSI values of the MVDR algorithm in the three types of weather data and the total data were higher than those of the CIDA algorithm, respectively. And all FAR values were lower than those of the CIDA algorithm, respectively.

(2) The effect of dealiasing velocity algorithm was related to the weather type of data. Among the three types of weather data, these two dealiasing algorithms both showed the best effect on heavy precipitation data. That is, among all of the three types of data, the two dealiasing algorithms achieved the highest POD value and CSI value, and the lowest FAR value in heavy precipitation data, because, compared with the other two types of data, the heavy precipitation data contained less strong wind shear and less isolated regions, which reduced the difficulty of dealiasing.

(3) For different types of weather data, the score difference between the two algorithms was quite different. For POD value and CSI value of heavy precipitation data, the MVDR algorithm was only $1.34 \%$ higher than CIDA algorithm. For typhoon data and squall line data, the POD value of the MVDR algorithm was 2.19\% and 5.66\% higher than that of CIDA algorithm, and the CSI value was 4.76\% and $6.29 \%$ higher than that of CIDA algorithm, respectively. The MVDR algorithm showed an advantage over the CIDA algorithm in the dealiasing effect of typhoons and squall lines.

The parameters of dealiasing methods can directly affect the results. So we should obtain the optimal values of the parameters and study their impact on performance. The parameters needed by the proposed algorithm include $G_{1}$ and $G_{2}$. Here, we used a grid search to find the optimal parameters. Grid search was a method that finds an optimal combination of parameters by exhaustive searching. Data from 13 events were used to maximize CSI values and determine the optimal parameters. According to our preliminary research, the candidate sets of parameters were as follows: $G_{1}=\left(1.1 V_{N}, 1.2 V_{N}, 1.3 V_{N}, 1.4 V_{N}, 1.5 V_{N}\right.$, $\left.1.6 V_{N}, 1.7 V_{N}\right)$ and $G_{2}=\left(1.2 V_{N}, 1.3 V_{N}, 1.4 V_{N}, 1.5 V_{N}\right.$, $\left.1.6 V_{N}\right)$. According to Table 2, when $G_{1}$ was in the range of $1.2 V_{N}-1.6 V_{N}$ and $G_{2}$ was in the range of $1.3 V_{N}-1.4 V_{N}$, the CSI values of the proposed algorithm were greater than or equal to $98.50 \%$. This showed that the MVDR algorithm had a good effect in a wide range of parameters $G_{1}$ and $G_{2}$.

When the radial velocity data was continuous, the CIDA algorithm and MVDR algorithm both worked very well. However, when there was a noise, shear, or invalid data around the aliased data, the dealiasing algorithms may fail.
TABLE 2: The changes of CSI values with parameters $G_{1}$ and $G_{2}$.

\begin{tabular}{|c|c|c|c|c|c|c|}
\hline & \multirow[b]{2}{*}{ CSI } & \multicolumn{5}{|c|}{$G_{2}$} \\
\hline & & $\begin{array}{c}1.2 V_{N} \\
(\%)\end{array}$ & $\begin{array}{c}1.3 V_{N} \\
(\%)\end{array}$ & $\begin{array}{c}1.4 V_{N} \\
(\%)\end{array}$ & $\begin{array}{c}1.5 V_{N} \\
(\%)\end{array}$ & $\begin{array}{c}1.6 V_{N} \\
(\%)\end{array}$ \\
\hline \multirow{7}{*}{$G_{1}$} & $1.1 V_{N}$ & 97.22 & 98.56 & 98.53 & 98.27 & 98.08 \\
\hline & $1.2 V_{N}$ & 97.12 & 98.66 & 98.63 & 98.45 & 98.17 \\
\hline & $1.3 V_{N}$ & 97.18 & 98.57 & 98.62 & 98.44 & 98.09 \\
\hline & $1.4 V_{N}$ & 97.16 & 98.58 & 98.54 & 98.44 & 98.15 \\
\hline & $1.5 V_{N}$ & 97.15 & 98.53 & 98.50 & 98.40 & 98.05 \\
\hline & $1.6 V_{N}$ & 97.04 & 98.54 & 98.50 & 98.33 & 98.05 \\
\hline & $1.7 V_{N}$ & 97.97 & 97.95 & 97.90 & 97.74 & 97.45 \\
\hline
\end{tabular}

Two results of both dealiasing algorithms were exemplified in Figures 7 and 8. Figure 7(a) showed the vector wind fields for the ERA-Interim results at $900 \mathrm{hPa}$ at 15:00 UTC on September 14, 2016. In Figure 7(a), there was a vortex and the center of the vortex was located about $80 \mathrm{~km}$ southeast of Xiamen radar station. And the scale of the velocity vectors was at the bottom left corner of Figure $7(a)$. Figure $7(b)$ showed the raw radial velocity field of the $0.5^{\circ}$ elevation observed by Xiamen radar at 14:59 UTC, September 14, 2016. According to the vector wind in Figure 7(b), there should be an amount of aliased data in the southeast direction of the radar station. These aliased data were manually dealiased shown in Figure 7(c), and they were marked by three white ellipses. Figures 7 (d) and 7(e) were the dealiasing results of the CIDA algorithm and MVDR algorithm, respectively. In the upper and lower regions marked by ellipses, both algorithms correctly dealiased. In the middle region marked by an ellipse, the CIDA algorithm failed to correct part of the aliased velocity due to missing data between the aliased velocity and the radar station. While the MVDR algorithm successfully dealiased due to the dealiasing between interregions. Same as Figure 7, Figure 8 showed another example of dealiasing. Figure 8(a) showed the vector wind fields for the ERA-Interim results at $500 \mathrm{hPa}$ at 18:00 UTC on May 13, 2016, and the southwest wind was the leading direction at that time. The scale of the velocity vectors was also at the bottom left corner of Figure $8(\mathrm{a})$. Figure $8(\mathrm{~b})$ showed the raw radial velocity dealiasing of the $2.4^{\circ}$ elevation velocity observed by Binzhou radar at 18:13 UTC on May 13, 2016. Thus, there was some aliased data in the upper left part of Figure 8(b). Figures $8(\mathrm{c})-8(\mathrm{e})$ showed the manually dealiased results marked by two white ellipses, the dealiasing results of the CIDA algorithm and the MVDR algorithm, respectively. It can be seen that the CIDA algorithm failed to correct part of the aliased velocity data on the upper ellipse because of the noise between this region and the radar station. And the CIDA algorithm also failed to dealiase on the lower side due to the lack of reference velocity. The dealiasing effect of the MVDR algorithm on the upper side shown in Figure 8(e) is better than that of the CIDA algorithm, because the step of intraregional dealiasing corrected the aliased velocities in the main velocity echo, and the aliased velocities in isolated velocity regions were dealiased by the step of interregional dealiasing. But our method also failed to correct the aliased 


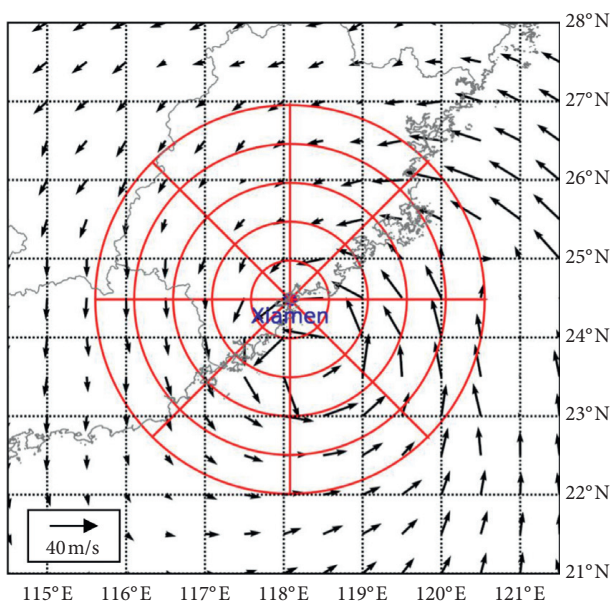

(a)

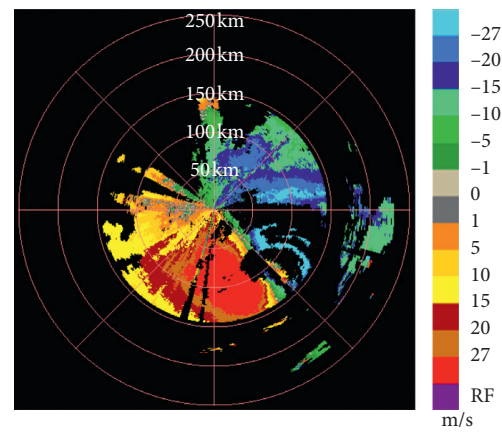

(d)
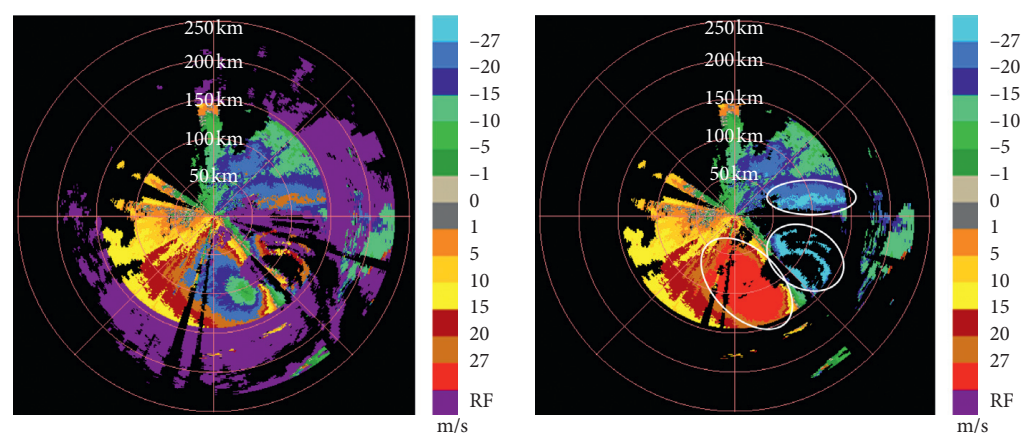

(b)

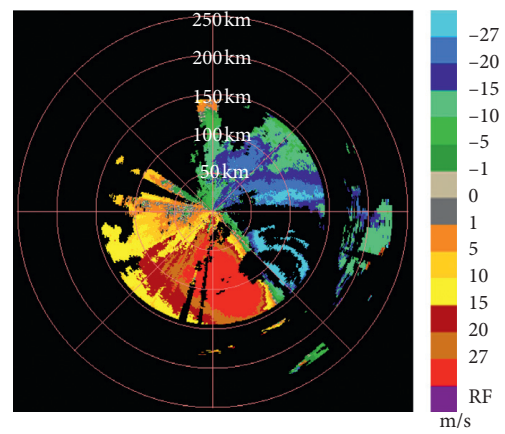

(e)

Figure 7: (a) The vector wind fields from the ERA-Interim at $900 \mathrm{hPa}$ at 15:00 UTC on September 14, 2016. The radial velocity field of $0.5^{\circ}$ elevation observed by Xiamen radar at 14:59 UTC, September 14, 2016: (b) raw velocity field, (c) manually dealiased velocity field, (d) dealiased velocity field by the CIDA algorithm, and (e) dealiased velocity field by the MVDR algorithm. The manual dealiased results are marked by white ellipses in (c).

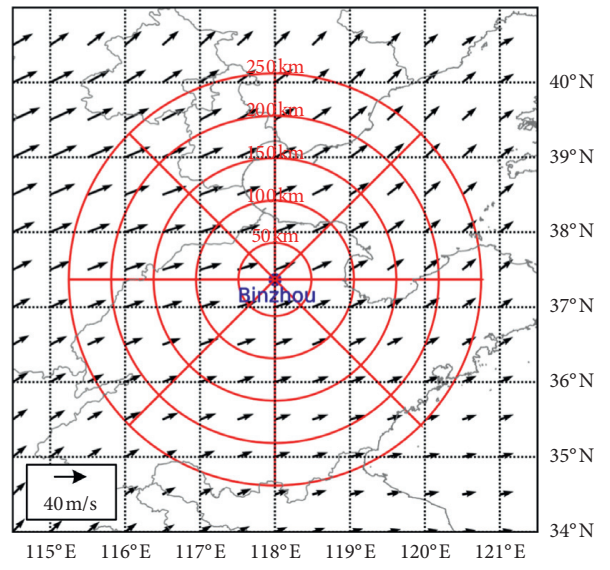

(a)

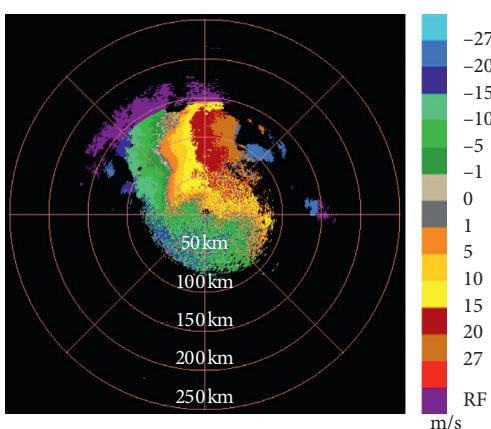

(b)

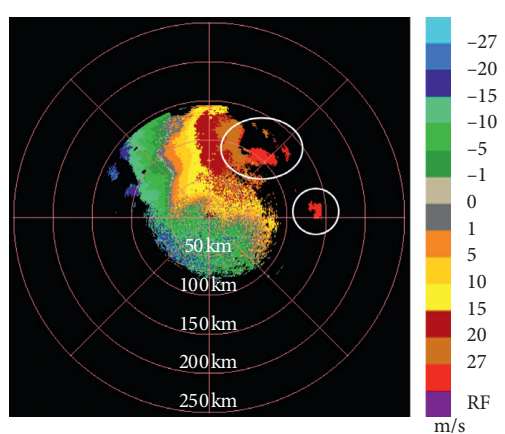

(c)

Figure 8: Continued. 


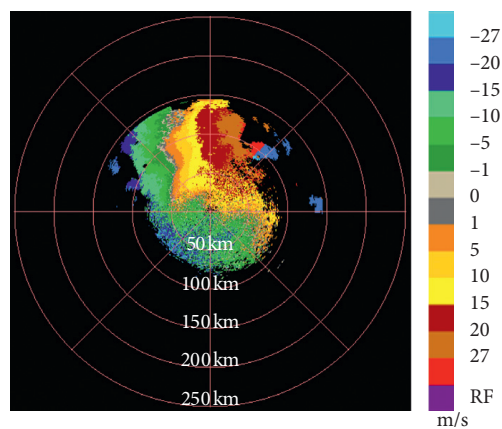

(d)

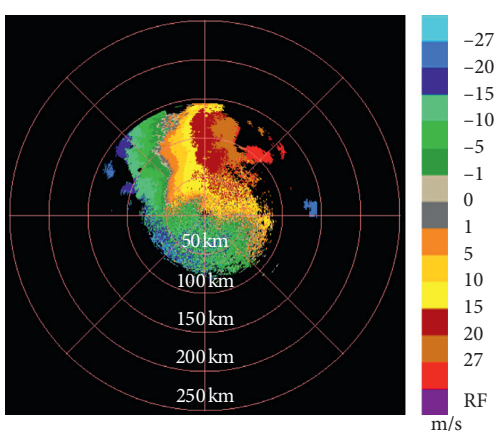

(e)

Figure 8: (a) The vector wind fields from the ERA-Interim at $500 \mathrm{hPa}$ at 18:00 UTC on May 13, 2016. The radial velocity field observed by Binzhou radar at 2.4 elevation angle at 18:13 UTC on May 13, 2016: (b) raw velocity field, (c) manually dealiased velocity field, (d) dealiased velocity field by the CIDA algorithm, and (e) dealiased velocity field by the MVDR algorithm. The manually dealiased results are marked by white ellipses in (c).

velocity data on the lower side. This was due to the small velocity difference between the velocity of the isolated aliased region on the lower side and the velocity of the boundary points of the adjacent region, which was wrongly judged as the strong wind shear. To sum up, the examples in Figures 7 and 8 gave some intuitive explanations of the main reasons for the success and failure of the CIDA algorithm and MVDR algorithm, respectively.

Our algorithm performed well on the test with 604 volume scans under three different types of weather. In the experiment, $98.87 \%$ of the aliased data were correctly dealiased. There were two types of errors in dealiasing. One type was the aliased data that was not correctly dealiased, which accounted for only $1.13 \%$ of the aliased data. The other type was that the normal data was incorrectly dealiased, and these data accounted for only about $0.35 \%$ of the dealiased data. To illustrate the extent of these errors, we evaluated the performance of our algorithm in another way. In the most problematic elevation of each volume scan, the average error data as a percentage of valid data was only $1.7 \%$.

We also made statistics on the causes of errors in dealiasing. Errors were caused by a variety of reasons. The most common reason (58.8\%) was noise. In some cases, a certain amount of noise would affect the calculation results of the sum of gradients in intraregional dealiasing or the sum of weights in interregional dealiasing, which would lead to errors. The other major reason (35.7\%) was the lack of valuable velocity reference points (e.g., Figure 8). A small number $(5.4 \%)$ of the errors was undetermined. The reference velocity in other elevations or in the previous volume scan for dealiasing may be used to solve these problems.

\section{Discussion and Conclusion}

Dealiasing is an important basic work in radar quality control. The dealiasing algorithm with high quality is helpful for wind retrieval, data assimilation, disaster automatic identification and monitoring, and strong convection nowcasting.
In this paper, a region-based method is proposed to dealiase velocity. The method involves two parts. The first part is dealiasing in every isolated region. By seeking the couples of points with the abnormal gradient in a region, subregion segmentation based on a couple of points with abnormal gradient is obtained, and dealiasing velocity in the region with rules is operated. It is an important feature of this algorithm to find the aliased velocities based on the sum of velocity gradients of the region. The second feature is to add the step of discrimination of abnormal gradients between points in a connected region. By setting a threshold value close to $2 V_{N}$, it can avoid or reduce the false dealiasing of strong wind shear regions. For region segmentation, the region growing method based on the gradient is used to cluster the weak shear region into a subregion. Compared with the method of dividing the close velocity into subregions, the number of subregions is reduced effectively in this way. Thus, the speed of this step can be improved. The second part is dealiasing in interregions, in order to solve the problem of the aliased velocities in the whole region. The key velocity points in adjacent regions are determined, and the velocity difference and their weights between adjacent regions are obtained. The weights containing distance and confidence information are constructed, and the aliased velocities are corrected according to the sum weights. Different from other methods, this paper makes use of the velocity, distance, and the confidence information of adjacent regions to determine whether the velocity in this region is aliased, which increases the anti-interference ability of the dealiasing velocity algorithm and improves the correction rate of the aliased velocities.

The MVDR algorithm also needs to obtain a valuable reference point of velocity in the radial velocity field. If this condition is not available, it is suggested to search for reference velocity around the corresponding region of different elevation angles up and down according to the space-time continuity of velocity, or even search for reference velocity in the previous volume scan. Therefore, the two-dimensional dealiasing velocity algorithm in this paper can be extended 
to three-dimensional, or even four-dimensional, for further improving the accuracy of the algorithm.

\section{Data Availability}

The Doppler weather radar data used to support the findings of this study may be released upon application to the China National Meteorological Information Centre, which can be contacted at http://data.cma.cn/.

\section{Conflicts of Interest}

The authors declare no conflicts of interest.

\section{Acknowledgments}

This study was partially supported by the Applied Foundation and Frontier Technology Research Program (Youth Project) of Tianjin, China (16JQNJC07500). The authors are thankful to China Meteorological Administration and Tianjin Meteorological Station for providing a lot of radar data and many manually checked results.

\section{References}

[1] R. J. Doviak, D. S. Zrnic, and R. M. Schotland, "Doppler radar and weather observations," Applied Optics, vol. 33, p. 4531, 1994.

[2] S. M. Torres, Y. F. Dubel, and D. S. Zrnić, "Design, implementation, and demonstration of a staggered PRT algorithm for the WSR-88D," Journal of Atmospheric and Oceanic Technology, vol. 21, no. 9, pp. 1389-1399, 2004.

[3] Y. Yu and Y. Li, "An improved clutter suppression method for weather radars using multiple pulse repetition time technique," Advances in Meteorology, vol. 2017, Article ID 8173643, 7 pages, 2017.

[4] Q. Xu, K. Nai, L. Wei, P. Zhang, S. Liu, and D. Parrish, “A VAD-based dealiasing method for radar velocity data quality control," Journal of Atmospheric and Oceanic Technology, vol. 28, no. 1, pp. 50-62, 2011.

[5] P. S. Ray and C. Ziegler, "De-aliasing first-moment Doppler estimates," Journal of Applied Meteorology, vol. 16, no. 5, pp. 563-564, 1977.

[6] D. Bargen and R. Brown, "Interactive radar velocity unfolding. preprints," in Proceedings of the 19th Conference on Radar Meteorology, Boston, MA, USA, 1980.

[7] M. Merritt, "Automatic velocity de-aliasing for real-time applications (in radar wind velocity measurement), " in Proceedings of the 22nd Conference on Radar Meteorology, Zurich, Switzerland, 1984.

[8] T. A. Boren, "An artificial intelligence approach to doppler weather radar velocity de-aliasing," Ph.D. thesis, University of Oklahoma, Norman, Oklahoma, 1986.

[9] W. R. Bergen and S. C. Albers, "Two- and three-dimensional de-aliasing of Doppler radar velocities," Journal of Atmospheric and Oceanic Technology, vol. 5, no. 2, pp. 305-319, 1988.

[10] M. D. Eilts and S. D. Smith, "Efficient dealiasing of Doppler velocities using local environment constraints," Journal of Atmospheric and Oceanic Technology, vol. 7, no. 1, pp. 118128, 1990.
[11] J. Gong, L. L. Wang, and Q. Xu, "A three-step dealiasing method for Doppler velocity data quality control," Journal of Atmospheric and Oceanic Technology, vol. 20, no. 12, pp. 1738-1748, 2003.

[12] J. Zhang and S. Wang, "An automated 2D multipass Doppler radar velocity dealiasing scheme," Journal of Atmospheric and Oceanic Technology, vol. 23, no. 9, pp. 1239-1248, 2006.

[13] A. Witt, R. Brown, and Z. Jing, "Performance of a new velocity dealiasing algorithm for the WSR-88D," in Proceedings of the 34th Conference on Radar Meteorology, Williamsburg, VA, USA, 2009.

[14] G. He, G. Li, X. Zou, and P. S. Ray, "Applications of a velocity dealiasing scheme to data from the China new generation weather radar system (CINRAD)," Weather and Forecasting, vol. 27, no. 1, pp. 218-230, 2012.

[15] Q. Xu and K. Nai, "A two-step variational method for analyzing severely aliased radar velocity observations with small Nyquist velocities," Quarterly Journal of the Royal Meteorological Society, vol. 139, no. 676, pp. 1904-1911, 2013.

[16] C. N. James and R. A. Houze Jr., "A real-time four-dimensional Doppler dealiasing scheme," Journal of Atmospheric and Oceanic Technology, vol. 18, no. 10, pp. 1674-1683, 2001.

[17] P. Tabary, G. Scialom, and U. Germann, "Real-time retrieval of the wind from aliased velocities measured by Doppler radars," Journal of Atmospheric and Oceanic Technology, vol. 18 , no. 6, pp. 875-882, 2001.

[18] Q. Xu, K. Nai, S. Liu, C. Karstens, T. Smith, and Q. Zhao, "Improved Doppler velocity dealiasing for radar data assimilation and storm-scale vortex detection," Advances in Meteorology, vol. 2013, Article ID 562386, 10 pages, 2013.

[19] Q. Xu and K. Nai, "Mesocyclone-targeted Doppler velocity dealiasing," Journal of Atmospheric and Oceanic Technology, vol. 34, no. 4, pp. 841-853, 2017.

[20] Z. Jing and G. Wiener, "Two-dimensional dealiasing of Doppler velocities," Journal of Atmospheric and Oceanic Technology, vol. 10, no. 6, pp. 798-808, 1993. 